\title{
Vulnerability study of myelinated and unmyelinated nerve fibers in acute ocular hypertension in rabbit
}

\author{
JIYING SHEN ${ }^{1,2}$, QIANG YANG ${ }^{2}$, DAOYI YU ${ }^{3}$, JIHONG WU ${ }^{2}$, YUANFANG ZHU ${ }^{2}$ and WENYI GUO ${ }^{2,4}$ \\ ${ }^{1}$ Department of Ophthalmology, Shanghai Peace Eye Hospital, Shanghai 200437; ${ }^{2}$ Department of Ophthalmology, \\ Eye \& ENT Hospital of Fudan University, Shanghai 200031, P.R. China; ${ }^{3}$ Centre for Ophthalmology \& Visual \\ Science, The University of Western Australia, Perth, WA 6009, Australia; ${ }^{4}$ Department of Ophthalmology, Shanghai \\ Ninth People's Hospital, Shanghai Jiaotong University School of Medicine, Shanghai 200011, P.R. China
}

Received September 18, 2016; Accepted July 11, 2017

DOI: $10.3892 / \mathrm{mmr} .2017 .7474$

\begin{abstract}
In the current study, it was aimed to evaluate the changes in myelinated and unmyelinated nerve fibers in retinal ischemia-reperfusion injuries caused by acute ocular hypertension and to determine the sequence of these changes. Adult healthy New Zealand white rabbits were randomized to the hemodynamic group [ $\mathrm{n}=12$; used to determine the optimal intraocular pressure (IOP) for the subsequent experiments] and the hypertension group $(\mathrm{n}=6 ; 70-\mathrm{mmHg}$ hypertension induced in one eye). IOP was adjusted using a cannula and saline. Doppler ultrasound was used to measure the velocity of the optic artery under different intraocular pressures. Immunohistochemistry for myelin basic protein (MBP) was performed. Apoptosis of retinal cells was detected by terminal deoxynucleotidyl transferase biotin-dUTP nick end labeling (TUNEL) assay. Electron microscopy was used to investigate the changes in myelinated and unmyelinated nerve fibers. IOP of the hypertension eyes was maintained at $70.2 \pm 1.0 \mathrm{mmHg}$, while IOP of control eyes was 7-14 mmHg. Doppler ultrasound demonstrated an obvious decline of peak systolic velocity and an increase of resistance index of retinal bloodstream under a 70-mmHg IOP. MBP immunohistochemistry and electron microscopy demonstrated obvious injuries to the myelin fibers. TUNEL indicated a significantly higher apoptosis rate in the hypertension eyes compared with control eyes. The apoptosis rate of retinal ganglion cells and bipolar cells in unmyelinated
\end{abstract}

Correspondence to: Dr Wenyi Guo, Department of Ophthalmology, Shanghai Ninth People's Hospital, Shanghai Jiaotong University School of Medicine, 639 Zhizaoju Road, Shanghai 200011, P.R. China E-mail: guowy975@sina.com

Abbreviations: IOP, intraocular pressure; MBP, myelin basic protein; RGCs, retinal ganglion cells; PSV, peak systolic velocity; RI, resistance index; IHC, immunohistochemistry; TUNEL, terminal deoxynucleotidyl transferase biotin-dUTP nick end labeling; SEM, scanning electron microscopy

Key words: rabbit, acute ocular hypertension, retinal ischemiareperfusion injury, myelin, glaucoma regions was higher than in myelinated regions. In conclusion, an IOP of $70 \mathrm{mmHg}$ led to incomplete retinal ischemia but was the threshold for retinal ischemia, leading to obvious injuries to the myelin fibers.

\section{Introduction}

Glaucoma is a lifelong-progressive irreversible blinding disease characterized by optic nerve damage and visual field defects. The World Health Organization listed it as the world's second most important cause of blindness, with 67 million people suffering from glaucoma worldwide (1). Binocular blindness caused by glaucoma accounts for $\sim 50 \%$ of the total number of the blind people, and $\geq 50 \%$ of patients with glaucoma are not diagnosed. The global prevalence is expected to reach 79.6 million people in 2020 (2).

The pathogenic factors that cause glaucoma vary, but only high intraocular pressure (IOP) is currently accepted as a main pathogenic factor (3). High IOP leads to a progressive and irreversible loss of retinal ganglion cells (RGCs), predominantly through two paths: i) Ischemia and hypoxia of the optic papilla and retina organization caused by a dramatically increasing IOP; and ii) retina ischemia-reperfusion injury occurring in the process of a rapid IOP decrease. Retina ischemia-reperfusion injury leads to degenerative damage of nerve cells caused by active inflammatory cells after a long history of ischemia and reperfusion of the retina (4).

Because of the unique structure and sieve axoplasmic transport and the theory stating that the cytoskeleton is involved in the structure and function of RGCs, optic nerve regeneration, and axoplasmic transport (5), the association between blood supply, IOP and the function of glial cells has not been widely investigated. Indeed, the optic fibers in the retina have no myelin, but when these unmyelinated nerve fibers come through the sieve plate, they are immediately wrapped by a myelin sheath to become myelinated nerve fibers. However, the role of the myelination of nerve fibers in glaucoma is still poorly understood.

Therefore, the current study used a rabbit model of ocular hypertension to simulate the pathological changes of acute glaucoma, understand the pathological changes in the different areas of the eye, to observe the characteristics of cell 
injury of the various layers of the retina and to understand the relationships between them. The results of this study may clarify the pathogenesis of glaucoma and provide theoretical evidence for novel preventive approaches and treatments of glaucoma.

\section{Materials and methods}

Animals. Healthy adult male New Zealand white rabbits $(\mathrm{n}=18)$ weighing 1,800-2,300 g were obtained from Yingen Rabbit Warren (Shanghai, China) and housed in specific pathogen-free conditions. Rabbits were maintained in a $12 \mathrm{~h}$ light/dark cycle environment with free access to food and water, temperature at $23 \pm 2^{\circ} \mathrm{C}$, and humidity at $60-70 \%$. They were randomized to the hemodynamic group $(n=12$; which was used to determine the optimal high IOP for the subsequent experiments) and the hypertension group $(\mathrm{n}=6 ; 70 \mathrm{mmHg}$ hypertension induced in one eye). The animals were treated in accordance with the Association for Research in Vision and Ophthalmology Statement for the Use of Animals in Ophthalmic and Vision Research (6) and the guidelines of the Committee on Animal Research of Fudan University (Shanghai, China). This study was approved by the ethics committee of Eye \& ENT Hospital of Fudan University.

Surgical procedures and model establishment. The rabbits were anesthetized with ketamine $(50 \mathrm{mg} / \mathrm{kg})$ and ILIUM XYLAZIL-100 (10 mg/kg). The rabbits were fixed on a frame with the right eye up. Two 25-gauge cannulae were inserted in the anterior chamber of the right eye. One cannula was connected to a pressure transducer (channel-1, PT1) and the other to a variable-pressure infusion of $0.9 \% \mathrm{NaCl}$ solution to monitor and adjust IOP. For the hemodynamic group, images were captured of the central retinal artery using a single-lens reflex camera connected to a surgical microscope. Color Doppler ultrasound was used to measure the ocular artery peak systolic velocity (PSV) and resistance index (RI) under different intraocular pressures (by increments of $10 \mathrm{mmHg}$ from $20-120 \mathrm{mmHg}$ ). For the hypertension group, the right eyes were used as the experimental eye and the left eyes were used as controls. Based on the initial experiments, an IOP of $70 \mathrm{mmHg}$ steadily maintained for $90 \mathrm{~min}$ was used in this group, with a reperfusion of 2 days. All rabbits were finally sacrificed with an anesthetic overdose. The eyes were immediately enucleated and stored in $10 \%$ formalin overnight at $4^{\circ} \mathrm{C}$ for 24-48 h. The eyes were cut along the equator. The posterior section was embedded in paraffin.

Immunohistochemistry (IHC) of myelin basic protein $(M B P)$. The six pairs of control and experimental eyes were sectioned $(4 \mu \mathrm{m})$ and the sections were flat-mounted on poly-L-lysine-coated slides. The sections were dewaxed and blocked with 5\% goat serum (G9023; Sigma-Aldrich; Merck KGaA, Darmstadt, Germany) diluted 1:20 in PBS, at room temperature for $20 \mathrm{~min}$, then exposed to mouse monoclonal anti-MBP (1:500; cat. no. ab24567; Abcam, Cambridge, MA, USA) prepared in PBS (0.1 M, pH 7.4) for $1.5 \mathrm{~h}$ at $37^{\circ} \mathrm{C}$. All specimens were subsequently given three washes over $15 \mathrm{~min}$ in a $4^{\circ} \mathrm{C}$ PBS wash solution. Slides were then incubated at room temperature with the secondary antibody: Supervision
Anti-Mouse Detection Reagent (undiluted; cat. no. D-3001; Kangwei Biotechnology, Co., Ltd., Beijing, China), for $30 \mathrm{~min}$. Specimens were washed three times over $15 \mathrm{~min}$ in a $4^{\circ} \mathrm{C}$ PBS wash solution and the staining was revealed using $0.05 \%$ diaminobenzidine at room temperature for $30 \mathrm{sec}$. Slides were subsequently mounted in neutral resin.

Myelin and retina were observed with an optical microscope at magnifications of x10 20, and 40. Images were captured with strictly controlled parameters. Images were analyzed using the Q500IW image analysis software (Leica Microsystems GmbH, Wetzlar, Germany). Images were transformed into black-and-white images. The light intensity was set to a range of 0-100; full penetration of the light was given a value of 100, whereas full light obstruction was given a value of 0 . The difference in light intensity between background and myelin was regarded as an indirect representation of the MBP content.

Terminal deoxynucleotidyl transferase biotin-dUTP nick end labeling (TUNEL) staining of retinal sections. Sections were stained using a TUNEL assay (TdT-FragEL DNA Fragmentation Detection Kit, Merck KGaA) (5) and counterstained with $3 \%$ methyl green at room temperature for $5 \mathrm{~min}$. Apoptotic cells were observed with a light microscope at a magnification of $\times 20$. Apoptotic cells were counted in three random fields.

Scanning electron microscopy (SEM). One piece of retinal tissue (1x5 mm) from the myelinated areas, unmyelinated area and macula were extracted, and ultra-thin sections cut $(2 \mu \mathrm{m})$. Following staining with $1 \%$ toluidine blue (preheated at $60^{\circ} \mathrm{C}$ ) at room temperature for $20 \mathrm{~min}$, sections were observed by SEM to observe the retinal ultrastructure.

Statistical analysis. SAS 8.2 (SAS Institute, Inc., Cary, NY, USA) was used for data analysis. Continuous data are expressed as the mean \pm standard deviation. The homogeneity of variance was tested. Groups were compared using analysis of variance with the Student-Newman-Keuls post hoc test. Two-sided $\mathrm{P}<0.05$ was considered to indicate a statistically significant difference.

\section{Results}

Determination of optimal high IOP of the hypertension model. The baseline IOP in the hypertension group was 7-14 mmHg. Rising to the $70-\mathrm{mmHg}$ target required $<30 \mathrm{sec}$, limiting the variations in IOP. The IOP was maintained stable at $70.2 \pm 1.0 \mathrm{mmHg}$ for $90 \mathrm{~min}$.

High IOP leads to retina ischemia. With the increase of IOP, a tapering of the diameter of the central retinal artery and vein supplying the myelinated nerve fibers was observed. The flow rate slowed down and the flow decreased, while choroidal vessels supplying the unmyelinated nerve fibers were closed. The optic papilla and the posterior pole retina became gradually plain, vessels became stiffer and vascular walls became thinner. When the intraocular pressure reached $70 \mathrm{mmHg}$, tissue ischemia was observed although a weak retinal vascular blood supply at the optic papilla with 


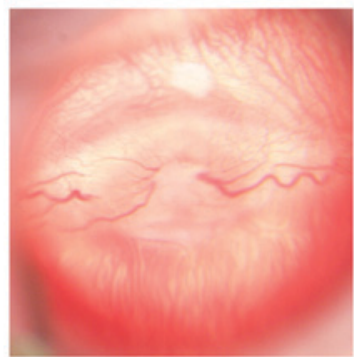

IOP $20 \mathrm{mmHg}$

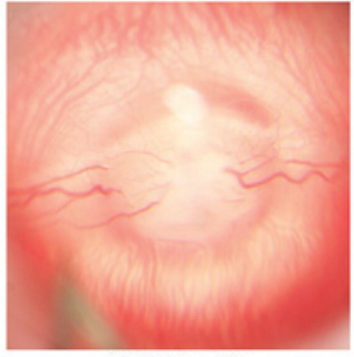

IOP $50 \mathrm{mmHg}$

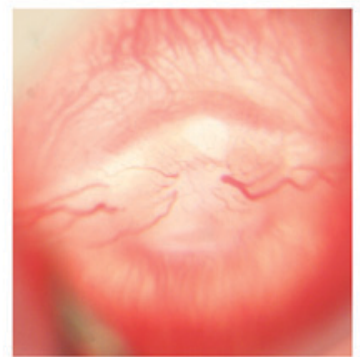

IOP $30 \mathrm{mmHg}$

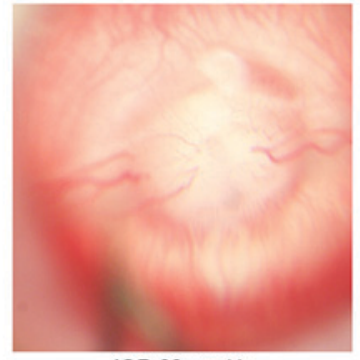

IOP $60 \mathrm{mmHg}$

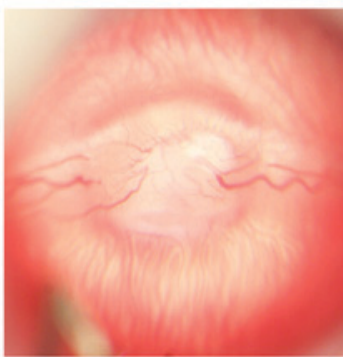

IOP $40 \mathrm{mmHg}$

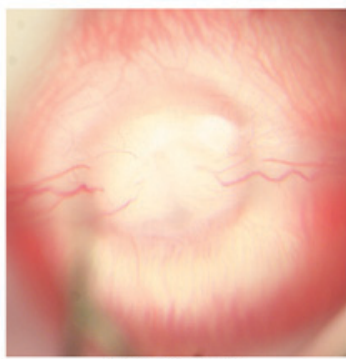

IOP $70 \mathrm{mmHg}$

Figure 1. High IOP led to retina ischemia. Increasing IOP from 20-70 mmHg in $10-\mathrm{mmHg}$ increments. With the increase of IOP, tapering of the diameter of the central retinal artery and vein supplying the myelinated nerve fibers was observed. The flow rate slowed down and the flow decreased, while choroidal vessels supplying the unmyelinated nerve fibers were closed. The optic papilla and the posterior pole retina became gradually plain, vessels became stiffer, and vascular walls became thinner. When the intraocular pressure reached $70 \mathrm{mmHg}$, tissue ischemia was observed although a weak retinal vascular blood supply at the optic papilla with fan-shaped capillary zone in the peripheral choroid were still visible. $\mathrm{n}=6$ per group. IOP, intraocular pressure.
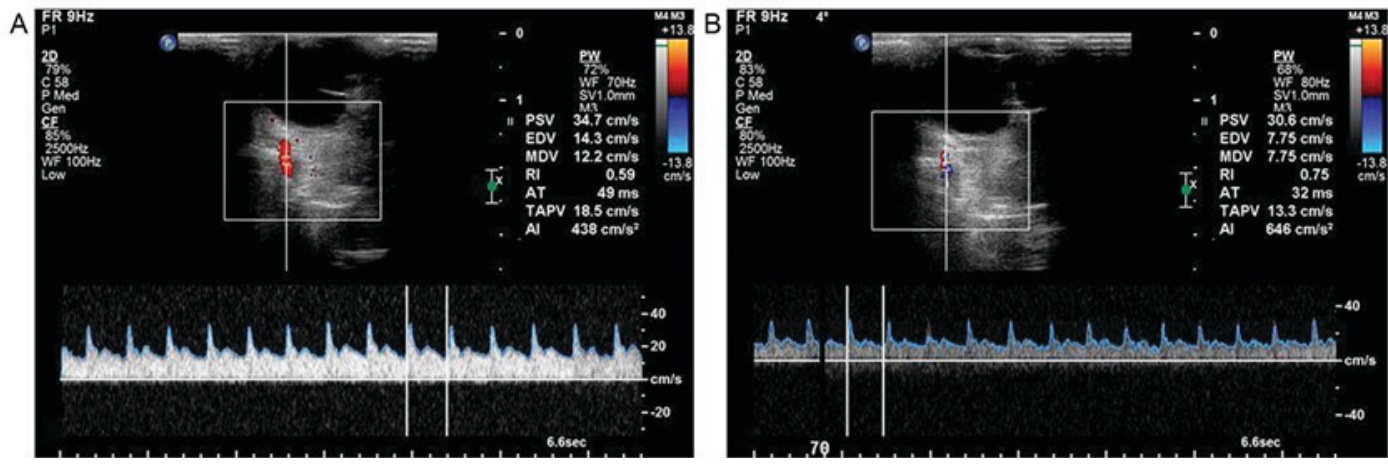

Figure 2. High IOP led to decreased retina blood flow according to Doppler ultrasound. The ophthalmic artery blood flow was fast, the artery had a thick diameter, and it displayed the characteristic sharp-angle waveform and a unique notch in the descending phase. (A) Retina without hypertension (7-14 mmHg). (B) Retina with IOP at $70 \mathrm{mmHg}$. $\mathrm{n}=6$ per group. IOP, intraocular pressure; PW, pulsed wave doppler; PSV, peak systolic velocity; EDV, end diastolic velocity; MDV, mean diastolic velocity; RI, resistive index.

fan-shaped capillary zone in the peripheral choroid was still visible (Fig. 1).

High IOP leads to decreased retina blood flow. Doppler ultrasound indicated that the retina was of moderate density, covered with many choroidal capillaries. The ophthalmic artery blood flow was fast, the artery had a thick diameter, and it displayed the characteristic sharp-angle waveform and a unique notch in the descending phase (Fig. 2).

With the increase of IOP, the number of opened capillaries decreased gradually. When the IOP reached $70 \mathrm{mmHg}$, there was no detectable flow on the retinal surface. In addition, ocular artery PSV decreased and RI increased gradually with increasing IOP. When IOP reached $70 \mathrm{mmHg}$, RI reached a peak but PSV was significantly decreased. There were significant differences between PSV at 20 and $70 \mathrm{mmHg}$, and RI between 20 and $70 \mathrm{mmHg}$ (data not show). Therefore,
$70 \mathrm{mmHg}$ was considered to be the threshold value for IOP in the acute ocular hypertension group.

High IOP increases MBP expression. The myelin tissue in the experimental eyes was stained dark brown and ordered in a fascicular manner, but the structure between fiber bundles was slightly loose, even cracked. The retinal tissue below was lightly stained, similar to the background, and had some differences compared with the myelin tissue. On the other hand, in control eyes, they were both lightly stained, almost the same as the background color. There were differences in light transmittance between the two groups of eyes (Fig. 3).

Two sets of MBP-stained sections were images at $\mathrm{x} 20$ and five fields were randomly selected in each image to determine the content of MBP (Fig. 4). The MBP expression level was higher in the high IOP eyes compared with control eyes $(77 \pm 9 \%$ vs. $65 \pm 9 \%$; $\mathrm{P}<0.001$; Table I). 


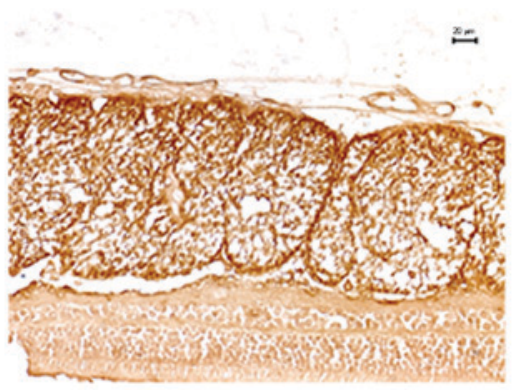

$\stackrel{\text { ma }}{=}$

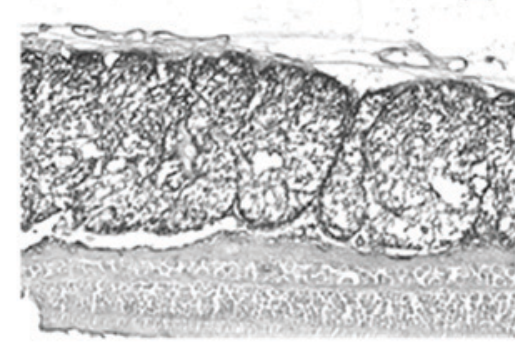

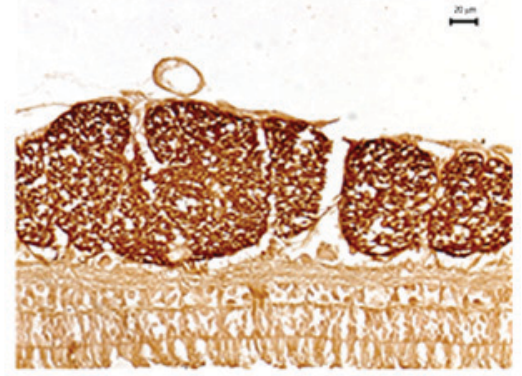

$\stackrel{x \rightarrow 0}{=}$

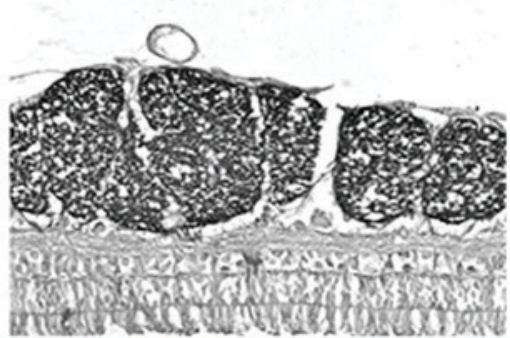

Figure 3. High IOP led to increased MBP expression. Myelin tissue was stained by anti-MBP immunohistochemistry in the (A) control eyes and (B) acute high IOP eye. The myelin tissue in the experimental eyes was stained dark brown and ordered in a fascicular manner, but the structure between the fiber bundles was slightly loose, even cracked. Retinal tissue was stained in control and high IOP eyes. The retinal tissue below was lightly stained, similar to the background, and had some differences compared to the myelin tissue in the experimental eyes. In the control eyes, the bundles were lightly stained, almost the same as the background color. There were differences in light transmittance between the two groups of eyes. $\mathrm{n}=6$ per group. IOP, intraocular pressure; MBP, myelin basic protein.
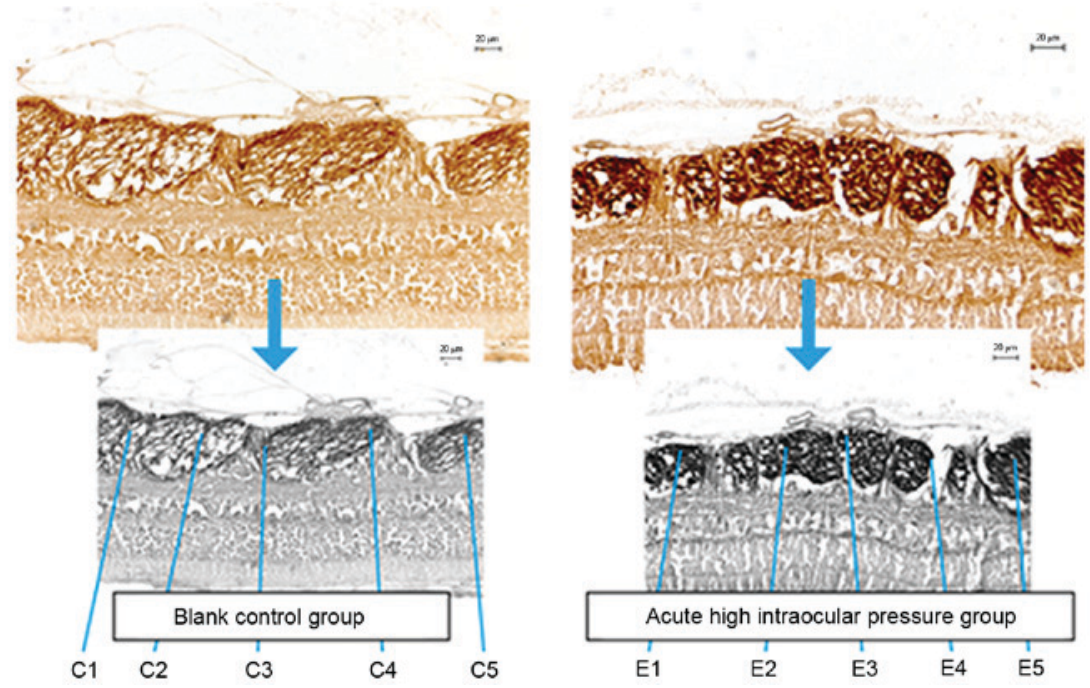

Figure 4. Semi-quantification of MBP expression. Two sets of MBP-stained sections were imaged at x20 magnification and five fields (C1-5 and E1-5) were randomly selected in each image to determine the content of MBP. MBP staining was increased in the eyes with intraocular pressure compared with controls.

High IOP led to increased retinal apoptosis. Fig. 5 presents representative TUNEL staining and Table II presents the related quantitative data. The apoptotic index of each cell layer of the high IOP eyes was higher than in the corresponding cell layer of the control group. In the high IOP eyes, the apoptotic index of each layer in the unmyelinated area was higher than in the myelinated area. These results were further analyzed using a factorial analysis of three repeated measurements (Table III). There was a difference in the cell apoptosis index in the different areas $(\mathrm{P}=0.003)$ and the difference was influenced by the groups. In the control group, the apoptosis rates of the myelinated and unmyelinated cells were similar. In the high IOP group, the cell apoptosis rate of the two areas was significantly different $(\mathrm{P}<0.0001)$, suggesting that the unmyelinated area was more easily injured than the myelinated area.

In addition, the cell apoptosis index among the three types of cells was also significantly different $(\mathrm{P}=0.018)$ and the difference was associated with the positions. Indeed, there were differences in the apoptosis rate of ganglion cells between the myelinated and unmyelinated areas. The apoptosis rate of the ganglion cells in unmyelinated area was higher than in myelinated area. This indicated that myelin has positive effects on ganglion cells apoptosis. 
Table I. Differences in myelin basic protein staining density between the experimental and control eyes.

A, Experimental eyes

Light transmittance

\begin{tabular}{lllllll}
\cline { 2 - 6 } Field & Rabbit 1 & Rabbit 2 & Rabbit 3 & Rabbit 4 & Rabbit 5 & Rabbit 6 \\
\hline 1 & 71.0843 & 91.0000 & 65.3846 & 92.0000 & 78.0000 & 71.2500 \\
2 & 75.9036 & 83.0000 & 62.0253 & 93.0000 & 77.0000 & 70.0000 \\
3 & 81.1765 & 85.0000 & 65.8228 & 93.0000 & 78.0000 & 67.5000 \\
4 & 73.2558 & 82.0000 & 60.0000 & 88.0000 & 86.0000 & 77.5000 \\
5 & 75.5814 & 85.0000 & 62.5000 & 78.0000 & 78.0000 & 72.5000 \\
\hline
\end{tabular}

B, Control eyes

Light transmittance

\begin{tabular}{lllllll}
\cline { 2 - 6 } Field & Rabbit 1 & Rabbit 2 & Rabbit 3 & Rabbit 4 & Rabbit 5 & Rabbit 6 \\
\hline 1 & 67.0455 & 78.0000 & 41.9753 & 73.0000 & 58.0000 & 59.2593 \\
2 & 67.7778 & 74.0000 & 56.6265 & 69.0000 & 57.0000 & 56.7901 \\
3 & 66.6667 & 74.0000 & 59.0361 & 79.0000 & 63.0000 & 56.0976 \\
4 & 69.6629 & 75.0000 & 56.6265 & 80.0000 & 64.0000 & 54.8780 \\
5 & 67.4157 & 68.0000 & 60.9756 & 75.0000 & 65.0000 & 51.2195 \\
\hline
\end{tabular}

$\mathrm{t}=5.2243 ; \mathrm{P}=2.4802 \mathrm{e}^{-6}$ experimental vs. control.
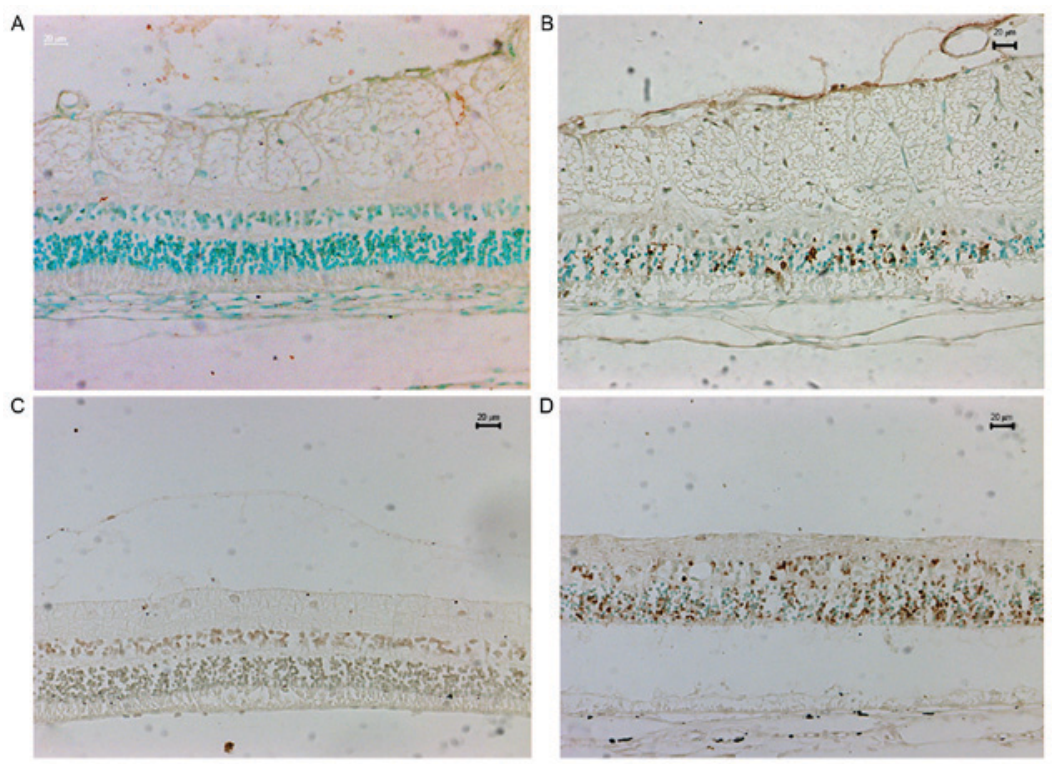

Figure 5. High IOP led to increased retinal apoptosis. Cell apoptosis of retinal structure of myelinated area of (A) control eyes and (B) high IOP eyes. Cell apoptosis of retinal structure of unmyelinated area in (C) control eyes and (D) high IOP eyes. $n=6$ per group. IOP, intraocular pressure.

A factorial analysis was performed for these three types of cells (Table IV). The injuries to ganglion and bipolar cell in the unmyelinated area were more severe than in the myelinated area, and the apoptosis index was also significantly different $(\mathrm{P}=0.04, \mathrm{RGC} ; \mathrm{P}=0.02$, bipolar cells), but there were no difference in apoptosis rate of photoreceptor cells between the unmyelinated and myelinated area.
High IOP changes the retinal ultrastructure. The ultrastructure of each layer of the experimental eyes with high IOP was injured to some extent (Fig. 6). A part of the axon was markedly swollen, forming a lacuna between the axon membrane and myelin sheath, with several cracks. Axoplasmic electron density was decreased and showed granular degeneration. The amounts of microtubules and microfilaments were reduced. 
Table II. Cell apoptosis index of different layers in the experimental and control eyes.

A, Unmyelinated

\begin{tabular}{lcccc}
\hline & \multicolumn{4}{c}{ Apoptosis index } \\
\cline { 2 - 5 } Cell layer & Mean & SD & Min & Max \\
\hline Control & & & & \\
RGC & 0.0278 & 0.0280 & 0.0000 & 0.2667 \\
Bipolar & 0.0179 & 0.0207 & 0.0000 & 0.0425 \\
$\quad$ Photoreceptor & 0.0042 & 0.0049 & 0.0000 & 0.0124 \\
Experimental & & & & \\
RGC & 0.7097 & 0.2064 & 0.4286 & 1.0000 \\
Bipolar & 0.5851 & 0.1530 & 0.4450 & 0.8508 \\
Photoreceptor & 0.4238 & 0.1647 & 0.1864 & 0.6636 \\
\hline
\end{tabular}

B, Myelinated

\begin{tabular}{lcccc}
\hline & \multicolumn{4}{c}{ Apoptosis index } \\
\cline { 2 - 5 } Cell layer & Mean & SD & Min & Max \\
\hline Control & & & & \\
RGC & 0.0278 & 0.0280 & 0.0000 & 0.1667 \\
Bipolar & 0.0060 & 0.0056 & 0.0000 & 0.0221 \\
Photoreceptor & 0.0052 & 0.0127 & 0.0000 & 0.0311 \\
Experimental & & & & \\
RGC & 0.3306 & 0.1887 & 0.0000 & 0.5000 \\
Bipolar & 0.3184 & 0.0795 & 0.2008 & 0.4243 \\
Photoreceptor & 0.3054 & 0.2195 & 0.0513 & 0.6585 \\
\hline
\end{tabular}

$\mathrm{n}=6$ per group. $\mathrm{SD}$, standard deviation; RGC, retinal ganglion cells.

Mitochondria were swollen and their membrane bilayer structure was unclear. Some cristae had disappeared and there was vacuolar degeneration. There were many mitochondrial vacuoles located in the lateral side of the ganglion cells. Bipolar cells exhibited edema, some with nucleus pyknosis and chromosome breakage. The appearance of photoreceptor cells of the outer nucleus layer was the same as bipolar cells (Fig. 6A). By contrast, in the control group, the ultrastructure of each layer was normal (Fig. 6B).

The structure of each layer of the myelinated area (Fig. 6C and D) was injured to a lesser extent than the unmyelinated area (Fig. 6A and B). Though bipolar cells and photoreceptors had lots of swelling and mitochondrial vacuolization, there were fewer signs of apoptosis such as nucleus pyknosis and chromosome breakage. By contrast, the injury of each layer of the unmyelinated area was more serious.

\section{Discussion}

The pathophysiological processes that cause glaucoma remain poorly defined. Thus, the current study aimed to evaluate the changes of myelinated and unmyelinated nerve fibers in retinal ischemia-reperfusion injuries caused by acute ocular hypertension and to determine the sequence of these changes. Doppler ultrasound showed an obviously decline of peak systolic velocity and an increase of resistance index of retinal bloodstream under an IOP of $70 \mathrm{mmHg}$. MBP IHC and electron microscopy demonstrated obvious injuries to the myelin fibers. TUNEL demonstrated a significantly higher apoptosis rate in the hypertension eyes compared with control eyes. The apoptosis rate of RGCs and bipolar cells in unmyelinated regions was higher than in myelinated regions. Thus, an IOP of $70 \mathrm{mmHg}$ led to an incomplete retinal ischemia, but was the threshold for retinal ischemia, leading to obvious injuries to the myelin fibers.

The observation of the capillaries on the retinal surface demonstrated that with increasing IOP more capillaries closed, decreasing blood supply. Coincidentally, when IOP reached $70 \mathrm{mmHg}$, Doppler ultrasound was unable to show the capillaries on the retinal surface, indicating retinal ischemia. Although it was not complete ischemia, it was enough to injure the retinal tissue. Early studies have demonstrated that the majority of patients with glaucomatous optic neuropathy and anterior ischemic optic neuropathy have retinal vascular autoregulation defects (7).

In addition to humans $(8,9)$, blood vessels of various other animals also undergo spontaneous adjustment when the pressure changes $(10,11)$. The central area of the optic papilla is the region across the intraocular papilla. When IOP reaches $40 \mathrm{mmHg}$, the blood flow is significantly reduced, and $40 \mathrm{mmHg}$ was speculated to be the threshold at which the central retinal artery can still autoregulate. When the vessel passes through the optic papilla and flows to the rim, the vascular autoregulation function is strengthened, the compressive range is widened, and it was necessary to increase IOP to $60-70 \mathrm{mmHg}$ to reduce the blood flow in the model of the present study.

The ciliary vascular system also has its own regulatory functions (12). The mean arterial pressure in rabbits is 70-75 mmHg (13). At this level, the IOP must be increased to $\sim 50 \mathrm{mmHg}$ in order to cause the ciliary blood flow to decrease (12). Similarly, the level of central retinal artery autoregulation capacity threshold is affected by the mean arterial pressure. Because the retinal blood flow depends on the ocular perfusion pressure, and because the ocular perfusion pressure is the difference of the mean arterial pressure and IOP, the increase or decrease of mean arterial pressure can directly affect the ocular perfusion pressure level and the blood supply. When IOP is increased to $70 \mathrm{mmHg}$, the ocular perfusion pressure is close to 0 , while the vast majority of retinal capillaries have no blood perfusion. In addition, because eye perfusion pressure is proportional to the mean arterial pressure and inversely proportional to the IOP, when the IOP is increased the cribriform plate anterior perfusion pressure decreases, but the screen plate perfusion pressure is not affected. Therefore, a huge pressure differential exists before and after the plate and is sufficient to threaten the blood flow and axoplasmic transport (14).

MBP is a flexible polypeptide located in the compact myelin and nucleus pulposus. Because of its specificity to oligodendrocytes, it is often used as a marker of oligodendrocytes, 
Table III. Factorial analysis of injury severity for retinal ischemia reperfusion of myelinated and unmyelinated areas.

\begin{tabular}{llcccrr}
\hline Times & \multicolumn{1}{c}{ Source } & DF & Type III SS & Mean square & F & P-value \\
\hline 1 & Group & 1 & 3.3389 & 3.3389 & 101.78 & $<0.0001$ \\
2 & Position & 1 & 0.3005 & 0.3005 & 15.27 & 0.0029 \\
& Position ${ }^{*}$ group & 1 & 0.2838 & 0.2838 & 14.42 & 0.0035 \\
3 & Cell & 2 & 0.0958 & 0.0479 & 2.85 & 0.0815 \\
& Cell ${ }^{*}$ group & 2 & 0.0534 & 0.0267 & 1.59 & 0.2291 \\
4 & Position ${ }^{*}$ cell & 2 & 0.0523 & 0.0261 & 4.97 & 0.0177 \\
& Position ${ }^{*}$ cell ${ }^{*}$ group & 2 & 0.0506 & 0.0253 & 4.82 & 0.0196 \\
\hline
\end{tabular}

DF, degrees of freedom; SS, sum of squares.

Table IV. Factorial analysis of injury severity and related factors of different retinal cells.

\begin{tabular}{|c|c|c|c|c|c|}
\hline Source & DF & Type III SS & Mean square & F & P-value \\
\hline \multicolumn{6}{|l|}{ Ganglion cells } \\
\hline Position & 1 & 0.2157 & 0.2157 & 5.32 & 0.0415 \\
\hline Group & 1 & 1.4545 & 1.4545 & 35.89 & $<0.0001$ \\
\hline ID (position) & 10 & 0.2072 & 0.0207 & 0.51 & 0.8498 \\
\hline \multicolumn{6}{|l|}{ Bipolar cells } \\
\hline Position & 1 & 0.1165 & 0.1165 & 7.40 & 0.0199 \\
\hline Group & 1 & 1.1606 & 1.1606 & 73.76 & $<0.0001$ \\
\hline ID (position) & 10 & 0.0756 & 0.0076 & 0.48 & 0.8708 \\
\hline \multicolumn{6}{|l|}{ Photoreceptors } \\
\hline Position & 1 & 0.0207 & 0.0207 & 1.09 & 0.3195 \\
\hline Group & 1 & 0.7772 & 0.7772 & 40.80 & $<0.0001$ \\
\hline ID (position) & 10 & 0.1894 & 0.0189 & 0.99 & 0.4996 \\
\hline \multicolumn{6}{|l|}{ Overall } \\
\hline Position & 1 & 0.3005 & 0.3005 & 4.23 & 0.0438 \\
\hline Cell & 2 & 0.0958 & 0.0479 & 0.67 & 0.5133 \\
\hline Position*cell & 2 & 0.0523 & 0.0261 & 0.37 & 0.6938 \\
\hline
\end{tabular}

DF, degrees of freedom; SS, sum of squares.

Schwann cells and Schwann cell tumors. Previous studies demonstrated that MBP has an important role in the developmental process of oligodendrocytes $(15,16)$. When the nerve tissue is subjected to ischemic injury or degenerative changes, the MBP is activated, thereby increasing synthesis and release, and its interstitial concentration rises (17). Therefore, it is considered an early specific indicator of disorders caused by exogenous factors or autoimmune neurological degenerative disorders (18). This study showed that acute ocular hypertension induced by ischemia reperfusion could lead to impairment of the myelin structure. In addition, SEM confirmed these damages.

The TUNEL cell apoptosis assay demonstrated that in ischemia reperfusion injury induced by acute ocular hypertension, the damage to unmyelinated nerve fibers was more severe than the damage to myelinated nerve fibers.
Myelin is a thick layer of protective film, and it can provide direct mechanical protection when exposed to high IOP. Additionally, rabbit eyes are similar to those of humans and their visual center is located in the unmyelinated area under the myelinated nerve fiber. Because the myelinated area is wrapped in myelin, retinal cells in the unmyelinated area apparently consume more oxygen and energy. Once the blood supply decreases, its tolerance to ischemia and hypoxia is correspondingly weaker (19).

The study of the rate of apoptosis for each layer demonstrated that ganglion cells and bipolar cells both followed the pattern of the apoptosis rate being higher in the unmyelinated area compared with the myelinated area; however, the photoreceptor cells did not exhibit the same pattern. Indeed, the photoreceptor cells are located in the outer layer, which is farther from the retinal vessels. The response of photoreceptor 


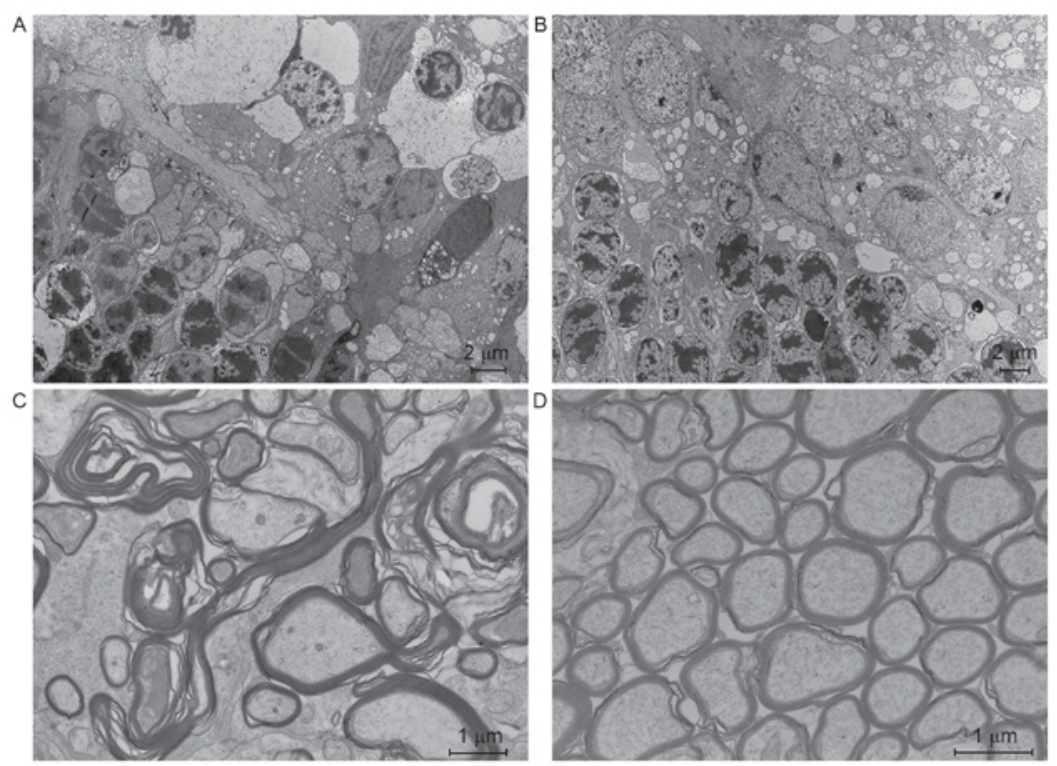

Figure 6. High intraocular pressure led to changes in the retinal ultrastructure. SEM micrographs of the retina of experimental and control eyes, (A) bipolar cells and photoreceptors of experimental eyes show vacuolization, nuclear pyknosis, and apoptosis, (B) morphology of bipolar cells and photoreceptors of control eyes is normal. SEM micrographs of myelinated nerve fibers, (C) myelin structure of experimental eye was loosely lamellar and axons showed mild degenerative changes, (D) myelin structure of control eyes was normal and the axons were not injured. $\mathrm{n}=6$ per group. SEM, scanning electron microscopy.

cells to the change of blood supply is slower, and they are less affected by ischemia reperfusion injuries (20). In addition, photoreceptor cells are located close to the sieve plate and an acutely elevated IOP can cause the sieve plate to sink, slowing down or even stopping the axoplasmic transport speed (21). On the other hand, ganglion and bipolar cells are far from the sieve plate, and the baxoplasmic transport distance is longer, making them more susceptible to changes in IOP. The deficiency of neurotrophic factors reduces their capacities of self-healing and resistance to ischemia, thereby increasing cell injury and eventually leading to irreversible cell apoptosis (19). When the retina is injured, the myelin area can synthesize a series of protective cytokines such as brain derived neurotrophic factor, neurotrophin-3 and insulin-like growth factor-1 (22). These cytokines can diffuse into the retina, increasing the resistance of cells to the injury, thus helping them to recover. Nevertheless, due to the anatomical structure, ganglion cells are certainly easier to be protected by these factors.

The present study is not without limitations. Of course, biases such as sample differences or sampling error and other factors, cannot be rules out. Additional studies remain necessary to address the complex question of the pathophysiology of glaucoma.

In conclusion, when the IOP reaches $70 \mathrm{mmHg}$, the rabbit retina is in a serious ischemic state and PSV drops, while RI peaks. Acute ocular hypertension leads to damage to the optic nerve myelin tissue and retinal cells of all layers, and the content of MBP in myelin was increased. TUNEL demonstrated that all layers of the retina had numerous apoptotic cells. Therefore, in acute ocular hypertension in rabbits, the main function of myelin may be to protect the optic nerve. This mechanism may be association to various factors and more comprehensive studies are required.

\section{Acknowledgements}

This work was supported by the National Natural Science Foundation of China (grant no. 30772372), the National High Technology Research and Development Program of China (863 Program) (grant no. 2008AA02Z422), the Science and Technology Commission of Shanghai (grant no. 14411960600) and the National Basic Research Program of China (973 Program) (grant no. 2007CB512204).

\section{References}

1. Quigley HA: Glaucoma. Lancet 377: 1367-1377, 2011.

2. Quigley HA and Broman AT: The number of people with glaucoma worldwide in 2010 and 2020. Br J Ophthalmol 90: 262-267, 2006.

3. Bengtsson B, Leske MC, Hyman L and Heijl A; Early Manifest Glaucoma Trial Group: Fluctuation of intraocular pressure and glaucoma progression in the early manifest glaucoma trial. Ophthalmology 114: 205-209, 2007.

4. Osborne NN, Casson RJ, Wood JP, Chidlow G, Graham M and Melena J: Retinal ischemia: Mechanisms of damage and potential therapeutic strategies. Prog Retin Eye Res 23: 91-147, 2004.

5. Reichstein D, Ren L, Filippopoulos T, Mittag T and Danias J: Apoptotic retinal ganglion cell death in the DBA/2 mouse model of glaucoma. Exp Eye Res 84: 13-21, 2007.

6. Romano PE: Association for research in vision and ophthalmology. Binocul Vis Strabismus Q 15: 358-359, 2000.

7. Flammer J and Mozaffarieh M: Autoregulation, a balancing act between supply and demand. Can J Ophthalmol 43: 317-321, 2008.

8. Pillunat LE, Anderson DR, Knighton RW, Joos KM and Feuer WJ: Autoregulation of human optic nerve head circulation in response to increased intraocular pressure. Exp Eye Res 64: 737-744, 1997.

9. Riva CE, Hero M, Titze P and Petrig B: Autoregulation of human optic nerve head blood flow in response to acute changes in ocular perfusion pressure. Graefes Arch Clin Exp Ophthalmol 235: 618-626, 1997.

10. Blumenröder S, Augustin AJ and Koch FH: The influence of intraocular pressure and systemic oxygen tension on the intravascular $\mathrm{pO} 2$ of the pig retina as measured with phosphorescence imaging. Surv Ophthalmol 42 (Suppl 1): S118-S126, 1997. 
11. Bouzas EA, Donati G and Pournaras CJ: Distribution and regulation of the optic nerve head tissue PO2. Surv Ophthalmol 42 (Suppl 1): S27-S34, 1997.

12. Nauli SM, Jin X and Hierck BP: The mechanosensory role of primary cilia in vascular hypertension. Int J Vasc Med 2011: 376281, 2011

13. Selbach MJ, Wonka F, Höper J and Funk RH: Effects of elevated intraocular pressure on haemoglobin oxygenation in the rabbit optic nerve head: A microendoscopical study. Exp Eye Res 69: 301-309, 1999.

14. Abbott CJ, Choe TE, Burgoyne CF, Cull G, Wang L and Fortune B: Comparison of retinal nerve fiber layer thickness in vivo and axonal transport after chronic intraocular pressure elevation in young versus older rats. PLoS One 9: e114546, 2014.

15. Galiano MR, Andrieux A, Deloulme JC, Bosc C, Schweitzer A, Job D and Hallak ME: Myelin basic protein functions as a microtubule stabilizing protein in differentiated oligodendrocytes. J Neurosci Res 84: 534-541, 2006.

16. Badan I, Buchhold B, Hamm A, Gratz M, Walker LC, Platt D, Kessler CH and Popa-Wagner A: Accelerated glial reactivity to stroke in aged rats correlates with reduced functional recovery. J Cereb Blood Flow Metab 23: 845-854, 2003.
17. Diekmann $\mathrm{H}$ and Fischer D: Glaucoma and optic nerve repair. Cell Tissue Res 353: 327-337, 2013.

18. Harauz G and Musse AA: A tale of two citrullines-structural and functional aspects of myelin basic protein deimination in health and disease. Neurochem Res 32: 137-158, 2007.

19. Guo WY, Cringle SJ, Su EN, Yu PK, Yu XB, Sun X, Morgan W and Yu DY: Structure and function of myelinated nerve fibers in the rabbit eye following ischemia/reperfusion injury. Curr Neurovasc Res 3: 55-65, 2006.

20. Ju WK, Kim KY, Hofmann HD, Kim IB, Lee MY, Oh SJ and Chun MH: Selective neuronal survival and upregulation of PCNA in the rat inner retina following transient ischemia. J Neuropathol Exp Neurol 59: 241-250, 2000.

21. Zhou J, Guo W, Fang Z, Yang Q, Wang Z and Yu D: The change of the optic nerve axoplasmic transport under different pressure state in acute high intraocular pressure model of rabbit. Zhong Hua Yan Di Bing Za Zhi Bian Ji Bu 27: 454-457, 2011 (In Chinese).

22. Du Y and Dreyfus CF: Oligodendrocytes as providers of growth factors. J Neurosci Res 68: 647-654, 2002. 